\title{
Electron nuclear double resonance study of photostimulated luminescence active centers in $\mathrm{CsBr}: \mathrm{Eu}^{2+}$ medical imaging plates
}

\author{
H. Vrielinck ${ }^{\mathrm{a}}$, F. Loncke ${ }^{\mathrm{a}}$, J.-P. Tahon ${ }^{\mathrm{b}}$, P. Leblans ${ }^{\mathrm{b}}$, P. Matthys ${ }^{\mathrm{a}}$ and F. Callens ${ }^{\mathrm{a}}$ \\ a Department of Solid State Sciences, Ghent University, Krijgslaan 281 - S1, B-9000 Gent, \\ Belgium \\ ${ }^{\mathrm{b}}$ Agfa HealthCare NV, Septestraat 27, B-2640 Mortsel, Belgium
}

\begin{abstract}
$\mathrm{CsBr}: \mathrm{Eu}^{2+}$ needle image plates (NIPs) exhibit an electron paramagnetic resonance (EPR) spectrum at room temperature (RT), whose intensity is correlated with the photostimulated luminescence (PSL) sensitivity of the plate. This EPR spectrum shows a strong temperature dependence : at RT it is due to a single $\mathrm{Eu}^{2+}(\mathrm{S}=7 / 2)$ center with axial symmetry, whereas at $\mathrm{T}<35 \mathrm{~K}$ the spectra can only be explained when two distinct centers are assumed to be present, a minority axial center and a majority center with nearly extremely rhombic symmetry. In this paper these low temperature centers are studied with electron nuclear double resonance (ENDOR) spectroscopy, which reveals the presence of ${ }^{1} \mathrm{H}$ nuclei close to the central $\mathrm{Eu}^{2+}$ ions in the centers. Analysis of the angular dependence of the ENDOR spectra allows to propose models for these centers, providing an explanation for the observed difference in intensity between the spectral components and for their temperature dependence.
\end{abstract}

\section{Introduction}

In radiography, digital image plates, based on X-ray storage phosphors, are in many hospitals rapidly replacing the conventional screen/film technology. X-ray irradiation produces room temperature (RT) stable defects, that store the image in the phosphor. This image can be read out via photostimulated luminescence (PSL) rendering the image in digital form [1]. The commonly used $\mathrm{BaFBr}: \mathrm{Eu}^{2+}$ powder based image plates, however, suffer from a loss of resolution due to light scattering. $\mathrm{CsBr}$, on the other hand, can be grown in needle image plates (NIPs). These are thermal vapour deposited binderless screens, consisting of oriented needle-shaped microcrystals, exhibiting a crystal $<100>$ direction perpendicular to 
the plate. Using NIPs strongly reduces the resolution problem and since $\mathrm{CsBr}: \mathrm{Eu}^{2+}$ has several other favorable X-ray storage properties (high X-ray absorption, high conversion efficiency, low read-out energy, easy erasability, ...) [2], it is not surprising that the appearance of $\mathrm{CsBr}: \mathrm{Eu}^{2+}$ based NIPs in hospitals is rapidly growing. The physics behind image storage and read-out in this phosphor are not yet well understood. For the $\mathrm{BaFBr}_{\mathrm{Eu}}{ }^{2+}$ phosphor, electron paramagnetic resonance (EPR) and electron nuclear double resonance (ENDOR) have proven to provide valuable information on the defects involved in these processes, as reviewed in Ref. [3].

In previous publications we reported the results of our Q band ( $34 \mathrm{GHz})$ EPR study on NIPs [4-6]. In as-deposited NIPs a strong EPR signal, labeled AA-EPR [4], was detected, in contrast with as-grown Bridgman $\mathrm{CsBr}: \mathrm{Eu}^{2+}$ single crystals, where no RT-stable EPR signal could be found ([7] and own results). Analysis of the RT spectrum showed it was due to a tetragonal $\mathrm{Eu}^{2+}$-related defect. Annealing the NIPs at $170-180^{\circ} \mathrm{C}$ for several hours proved to enhance both the intensity of this spectrum and the PSL sensitivity of the plate. Furthermore, a linear correlation was found between the intensity of the EPR signal of unexposed NIPs and the PSL yield after X-ray irradiation [5]. This strongly suggests that the Eu-related defect producing the AA-EPR spectrum plays a direct role in the storage (e.g. as a hole trap) and/or read-out (e.g. as the PSL active emission center) process of the image plate. Lowering the temperature proved to lead to drastic changes in the EPR spectrum. From $\mathrm{T}<$ $35 \mathrm{~K}$ onwards, the spectrum can only be explained assuming that two distinct $\mathrm{Eu}^{2+}$-related defects are present [6] : a minority defect with tetragonal symmetry giving rise to the AAEPR I spectrum, and a (nearly) extremely rhombic majority defect associated with the AAEPR II spectrum. For all these defects, the principal axes are found along crystallographic $<100>$ orientations. Following its temperature dependence in the $4-300 \mathrm{~K}$ range, the spectrum appears to undergo gradual changes, suggesting that a dynamical distortion present at RT slows down and eventually gets frozen out at lower temperatures. Finally, we also demonstrated that the "AA-EPR type" defects, stable at RT in NIPs, are fundamentally different from the $\mathrm{Eu}^{2+}$-related centers produced after heating $\mathrm{CsBr}$ :Eu single crystals (or NIPs [6]) to $500-600^{\circ} \mathrm{C}$ and rapid quench to $77 \mathrm{~K}$. The latter defects were reported by Savel'ev et al. [8] to aggregate at RT, and are most probably very similar to the $\mathrm{Eu}^{2+}$ defects associated with a cation vacancy found in other alkali halides (see e.g. [9] and references therein).

The detailed EPR analysis, however, did not allow us to propose a conclusive model for the AA-EPR type centers. For this reason ENDOR experiments were also undertaken and 
in our previous publication [6], we already pointed out the unexpected detection of ${ }^{1} \mathrm{H}$ transitions in these spectra. The present paper is devoted to the analysis of the ENDOR spectra (Section 3), with special emphasis on interactions with ${ }^{1} \mathrm{H}$ nuclei, which are indicative of $\mathrm{H}_{2} \mathrm{O}$ or $\mathrm{OH}^{-}$molecules in the close vicinity of the $\mathrm{Eu}^{2+}$ central paramagnetic ion of the defects. In Section 4, a model for the AA-EPR type centers is proposed, which in addition to the ${ }^{1} \mathrm{H}$ ENDOR transitions also explains the observed difference in intensity between AAEPR I and AA-EPR II and the temperature dependence of the spectra.

\section{Experimental}

\subsection{Samples}

EPR and ENDOR experiments were performed on a $1 \mathrm{~mm}$ thick $\mathrm{CsBr}: \mathrm{Eu}^{2+} \mathrm{NIP}$ prepared by Agfa-HealthCare NV via thermal vapour deposition and optimized for PSL activity, and on a commercial Hamamatsu plate. More details about these samples are given in Refs. $[5,6]$. $\mathrm{CsBr}$, as $\mathrm{CsCl}$, has a simple cubic lattice in which all $\mathrm{Cs}^{+}$ions are surrounded by $8 \mathrm{Br}^{-}$ions at the corners of a cube, and vice versa. XRD measurements revealed that the long axis of the needles corresponds to a $<100>$ crystal axis. As a result a single-crystal like $<100>$ spectrum can be recorded when the external magnetic field is perpendicular to the plate (or parallel to all needle axes). Rectangular pieces $\left(\sim 1.4\right.$ x $\left.10 \mathrm{~mm}^{2}\right)$ were carefully cut from the plates with a cleaving knife and positioned in a standard EPR (clear fused quartz) tube with the plane of the plate parallel to the axis of the sample tube.

\subsection{Spectrometry}

Q band EPR and ENDOR spectra were recorded with a Bruker ElexSys E500 spectrometer equipped with an Oxford CF935 flow cryostat. ENDOR spectra could only be recorded in the $3-4 \mathrm{~K}$ range. These temperatures were attained and stabilised by lowering the pressure in the He volume of the cryostat with a Sogevac SG40 rotary vane pump. In Ref. [6] we noted that the EPR spectral components rapidly broaden when the magnetic field is rotated away from the needle $<100>$ axes, but that nevertheless a complete angular dependence of the EPR spectrum could be recorded and analyzed. This broadening had, however, a more pronounced negative effect on the quality of the ENDOR spectra and as a result, ENDOR spectra could only be recorded within $20^{\circ}$ from the needle $<100>$ direction.

\subsection{Spectrum analysis}


The spectra were analyzed and simulated (including temperature effects) using the EasySpin routines [10]. $\mathrm{Eu}^{2+}$ has a $[\mathrm{Xe}] 4 \mathrm{f}^{7}$ configuration, resulting in an ${ }^{8} \mathrm{~S}_{7 / 2}$ ground state and an electronic spin $S=7 / 2$. Both ${ }^{151} \mathrm{Eu}$ and ${ }^{153} \mathrm{Eu}$ have $\mathrm{I}=5 / 2$ with natural abundances of $47.81 \%$ and $52.19 \%$, respectively. ${ }^{1} \mathrm{H}$ nuclei have $\mathrm{I}=1 / 2$. The ENDOR spectra for each nucleus were analyzed using a spin Hamiltonian describing the interaction of the unpaired electrons with a single nucleus :

$$
\hat{\mathrm{H}}=\mu_{\mathrm{B}} \overrightarrow{\mathrm{B}} \cdot \overrightarrow{\mathrm{g}} \cdot \hat{\overrightarrow{\mathrm{S}}}+\hat{\mathrm{H}}_{\mathrm{ZTS}}+\hat{\overrightarrow{\mathrm{S}}} \cdot \overrightarrow{\mathrm{A}} \cdot \hat{\overrightarrow{\mathrm{I}}}-\mathrm{g}_{\mathrm{N}} \mu_{\mathrm{N}} \overrightarrow{\mathrm{B}} \cdot \hat{\overrightarrow{\mathrm{I}}}+\hat{\overrightarrow{\mathrm{I}}} \cdot \overrightarrow{\mathrm{Q}}_{\mathrm{i}} \cdot \hat{\overrightarrow{\mathrm{I}}}
$$

including, respectively, the electronic Zeeman term, the zero-field splitting (ZFS) term, the hyperfine (HF) interaction, nuclear Zeeman term and quadrupole term, which only occurs for nuclei with $\mathrm{I}>1 / 2 . \mu_{\mathrm{B}}$ and $\mu_{\mathrm{N}}$ represent the Bohr and nuclear magneton, $\mathrm{g}_{\mathrm{N}}$ is the nuclear $\mathrm{g}$ factor for the interacting nucleus. The $\mathrm{g}$ tensor and the $\mathrm{B}_{\mathrm{k}}^{\mathrm{q}}$ parameters of the ZFS Hamiltonian, $\hat{\mathrm{H}}_{\mathrm{ZFS}}=\sum_{\mathrm{k}=2,4,6|q| \leq k} \sum_{\mathrm{k}} \mathrm{B}_{\mathrm{q}}^{\mathrm{q}} \hat{\mathrm{O}}_{\mathrm{k}}^{\mathrm{q}}$, with $\hat{\mathrm{O}}_{\mathrm{k}}^{\mathrm{q}}$ the extended Stevens operators (see e.g. [10] or [11]), for the RT AA-EPR spectrum and the low temperature AA-EPR I and AA-EPR II spectra have been determined in our previous EPR studies [4,6]. Their values, as determined in Refs. [4,6], are given in Table 1. In the principal axes frame of the defect, the second order terms of the ZFS Hamiltonian reduce to

$$
\begin{gathered}
\mathrm{B}_{2}^{0} \hat{\mathrm{O}}_{2}^{0}+\mathrm{B}_{2}^{2} \hat{\mathrm{O}}_{2}^{2}=\mathrm{D}_{\mathrm{x}} \mathrm{S}_{\mathrm{x}}^{2}+\mathrm{D}_{\mathrm{y}} \mathrm{S}_{\mathrm{y}}^{2}+\mathrm{D}_{\mathrm{z}} \mathrm{S}_{\mathrm{z}}^{2}=\hat{\overrightarrow{\mathrm{S}}} \cdot \overrightarrow{\mathrm{D}} \cdot \hat{\overrightarrow{\mathrm{S}}} \\
\mathrm{D}_{\mathrm{x}}=-\mathrm{B}_{2}^{0}+\mathrm{B}_{2}^{2} \quad \mathrm{D}_{\mathrm{y}}=-\mathrm{B}_{2}^{0}-\mathrm{B}_{2}^{2} \quad \mathrm{D}_{\mathrm{z}}=2 \mathrm{~B}_{2}^{0}
\end{gathered}
$$

For all defects under study here, the principal axes of the D tensor were, within experimental error, found along $<100>$ directions. In the following, the $x, y$ and $z$-axes of the centers/defects or components of the spectra refer to these $D_{x}, D_{y}$ and $D_{z}$ axes/components, respectively.

In order to clarify the description and interpretation of the ENDOR spectra, we here briefly repeat the analysis of the EPR spectra of the NIPs. The AA-EPR spectrum, recorded at RT with the magnetic field along the needle axes (B // <100>), is shown in Fig. 1a. For this magnetic field orientation, the defect can have its fourfold $<100>$ symmetry axis parallel (zoriented defect) or perpendicular (x or y-oriented) to the external field. The positions marked by arrows indicate the parallel and perpendicular features of AA-EPR, as calculated using the parameters in Table 1. The spectrum recorded at $4 \mathrm{~K}$, displayed in Fig. 1b, contains contributions of AA-EPR I, from a tetragonal center (parallel, $\mathrm{z}$ and perpendicular, $\mathrm{xy}$ components) and of AA-EPR II, due to an orthorhombic center (distinct $\mathrm{x}, \mathrm{y}$ and $\mathrm{z}$ - 
components). In all plates we investigated so far, the AA-EPR I and AA-EPR II spectra exhibit roughly the same intensity ratio, AA-EPR II being the dominant contribution.

In order to identify the interacting nuclei via their nuclear Zeeman interaction and to facilitate the interpretation of the ENDOR spectra for centers with overlapping EPR spectra with high electron and nuclear spin, we recorded the field dependence of the ENDOR spectrum through various EPR transitions in Fig. 1b. In Ref. [12] we have shown how these spectra in field - frequency space allow to identify the electronic and nuclear states involved in EPR and ENDOR transitions. In accordance with Ref. [13] we will refer to this type of spectra as field-frequency ENDOR (FF-ENDOR) spectra.

\section{ENDOR results}

\subsection{Europium interaction}

In Fig. 2a the FF-ENDOR spectrum recorded at $3.5 \mathrm{~K}$ with the magnetic field along the needle axes in the $1150-1250 \mathrm{mT}$ range is shown. Traces b and c of Fig. 2 show the experimental EPR spectrum in this magnetic field range and the simulation for the $\mathrm{x}$ component of the AA-EPR II spectrum, which dominates the spectrum in this range. The yand $\mathrm{z}$-component of this spectrum only contribute to the central line at $\sim 1200 \mathrm{mT}$. As an indication for the quality of individual ENDOR spectra, the spectrum at B $=1200 \mathrm{mT}$, marked by the dashed-dotted line in Fig. 2a is shown in trace d.

The most intense transitions in the FF-ENDOR spectrum (Fig. 2a) occur in the $40-60$ $\mathrm{MHz}$ range and exhibit a strong linear dependence on the magnetic field. As will be demonstrated in Section 3.2, these originate from ${ }^{1} \mathrm{H}$ nuclei. The next most intense transitions occur in the high-frequency range of $100-150 \mathrm{MHz}$ and have a much smaller linear field dependence. They occur as two nearly identical five-lines ENDOR patterns found around central field positions of 1170 and $1200 \mathrm{mT}$ and are marked by dashed ellipses. For both natural $\mathrm{Eu}$ isotopes, five-lines patterns are expected in the ENDOR spectra, but in view of the large hyperfine (HF) splitting, which is resolved in the EPR spectrum, none of the ENDOR spectra recorded at just one magnetic field position contains all five. Only in the FF-ENDOR spectrum, these fingerprints of interactions with $\mathrm{I}=5 / 2$ nuclei are easily recognized.

The fact that the transitions in the $100-150 \mathrm{MHz}$ range can be observed at $1170 \mathrm{mT}$ is a strong indication that they are connected with the x-component of the AA-EPR II spectrum, the only spectral component with appreciable EPR intensity in this range. The self

$\mathrm{HF}$ interaction for $\mathrm{Eu}^{2+}$ centers is relatively independent of the lattice surroundings $\left(\mathrm{A}\left({ }^{151} \mathrm{Eu}\right)\right.$ 
$\sim-95 \mathrm{MHz}$, see e.g. [9]). Hence, the transitions can be identified as those for the ${ }^{151} \mathrm{Eu}$ isotope within the $M_{S}=-3 / 2$ multiplet. Fitting the positions of the five lines, one finds $A_{x}\left({ }^{151} E u\right)=-$ 93.5 $\mathrm{MHz}$ and $\mathrm{Q}_{\mathrm{x}}\left({ }^{151} \mathrm{Eu}\right)=+2.87 \mathrm{MHz}$. Figure 2e shows an FF-ENDOR spectrum simulation for all components of the AA-EPR II spectrum, including contributions of both Eu isotopes, using the tabulated ratios for their magnetic and quadrupole moments $\left(\mathrm{g}_{\mathrm{N}}\left({ }^{153} \mathrm{Eu}\right) / \mathrm{g}_{\mathrm{N}}{ }^{151} \mathrm{Eu}\right)=$ $\left.0.4416 ; \mathrm{Q}\left({ }^{153} \mathrm{Eu}\right) / \mathrm{Q}\left({ }^{151} \mathrm{Eu}\right)=2.6711[10]\right)$. The asymmetric spacing within the five-lines patterns could only be obtained assuming the other components of the $\mathrm{Q}$ tensor to be large $\left(\mathrm{Q}_{\mathrm{y} / \mathrm{z}}\left({ }^{151} \mathrm{Eu}\right)=+5.32 \mathrm{MHz}, \mathrm{Q}_{\mathrm{z} / \mathrm{y}}\left({ }^{151} \mathrm{Eu}\right)=-8.32 \mathrm{MHz}\right)$. The simulation shows that practically all high frequency transitions in Fig. 2a are explained by the self HF interaction of AA-EPR II. Only transitions within highly populated $\mathrm{M}_{\mathrm{S}}$ multiplets are well observed, lending explicit support to the sign assignations for the ZFS parameters as determined by low temperature $\mathrm{W}$ band measurements [6]. Indeed, the other intense spots marked in Figs. 2a and e are ${ }^{153} \mathrm{Eu}$ transitions within the low lying $\mathrm{M}_{\mathrm{S}}=-7 / 2$ and $-5 / 2$ multiplets. For $\mathrm{Eu}^{2+}$ ions the self $\mathrm{HF}$ interaction is quite insensitive to the surroundings and the quadrupole interaction also only gives indirect information on the lattice environment. As the measurements were very time and liquid He consuming, no attempts were made to complete the self HF analysis for AAEPR II and AA-EPR I.

\subsection{Hydrogen interaction}

It is striking that the transitions around $50 \mathrm{MHz}$, which show a strong fielddependence, are not accounted for by the simulations in Fig. 2. Figure 3 shows a zoom of this region, on which also the ${ }^{1} \mathrm{H}$ Larmor frequency is indicated. For ${ }^{1} \mathrm{H}$ nuclei, exhibiting a small $\mathrm{HF}$ interaction with the unpaired electrons of $\mathrm{Eu}^{2+}$, pairs of ENDOR transitions in the vicinity of this Larmor frequency and with the same field dependence are expected (see e.g. Ref. [14]). Quite a few lines in the FF-ENDOR spectrum indeed satisfy these conditions. As this region of the EPR spectrum is multi-composite, we started the analysis of the hydrogen interaction for spectra recorded on well-isolated EPR transitions at lower and higher fields.

Figure 4 shows a selection of ENDOR spectra recorded on the AA-EPR I spectrum. As mentioned in Section 2, ENDOR spectra could only be recorded for magnetic field orientations sufficiently close (within $20^{\circ}$ ) to the needle axes (direction defined as $0^{\circ}$ ). When at $0^{\circ}$ the magnetic field is set to the $\mathrm{M}_{\mathrm{S}}:-7 / 2 \rightarrow-5 / 2$ transition of the $\mathrm{z}$-component of AAEPR I at $580 \mathrm{mT}$ (upper trace of Fig. 4a), two ENDOR transitions are observed split by $|\mathrm{A}|$ 4.7 MHz. From the spin Hamiltonian (1) one can calculate to first order that the ENDOR transition frequencies are expected at 


$$
v\left(M_{S}\right)=v_{L}-A_{S}
$$

At $580 \mathrm{mT}, v_{\mathrm{L}}=24.6 \mathrm{MHz}$ and the two observed ENDOR lines satisfy Eq. (3) if one assumes A to be positive. The spectrum at $0^{\circ}$ in Fig. $4 \mathrm{~b}$ at $1407 \mathrm{mT}$, corresponding to the $\mathrm{M}_{\mathrm{S}}:-5 / 2 \rightarrow$ $-3 / 2$ transition of the $\mathrm{x}=\mathrm{y}$ component, again contains only two intense lines, split by $|\mathrm{A}| \sim$ 2.2 MHz. At this magnetic field $v_{\mathrm{L}}=59.9 \mathrm{MHz}$ and the observed transition frequencies match Eq. (3) when A is negative. In Fig. 4, and also for all other ENDOR spectra recorded for AAEPR I, one observes that the transitions additionally split in two components when the magnetic field is rotated away from the $<100>$ needle axes. This type of angular dependence is characteristic for a HF tensor displaying so-called monoclinic-I symmetry, i.e. having three distinct principal values and one principal direction along a $<110>$ orientation. This is demonstrated in Fig. 5, where a simulation of the angular dependence of the ENDOR transitions following a single EPR line $\left(\mathrm{M}_{\mathrm{S}}:-5 / 2 \rightarrow-3 / 2\right)$ for two defect orientations $(\mathrm{z} \rightarrow \mathrm{x}$ and $\mathrm{x} \rightarrow \mathrm{y}$ ) is shown. For this simulation, the HF tensor in Table 2 is used, which is obtained by least-squares error fitting calculated to experimental ENDOR spectra. The simulations in Fig. 4 (red lines) are also performed using this tensor. The overall agreement is very satisfactory, especially if one considers that, because of the needle structure of the NIPs, when tilting the magnetic field away from $0^{\circ}$, the ENDOR spectra are in fact convolutions over many single crystal orientations, whereas the simulations are performed for only one orientation.

The ${ }^{1} \mathrm{H}$ HF tensor determined for AA-EPR I indicates that the interaction has a dominant (point) dipolar contribution. It is nearly traceless, has one large positive principal value $\left(A_{z}\right)$ and two nearly equal negative principal values $\left(A_{x} \approx A_{y}\right)$ with approximately half the coupling strength. For a pure point dipole interaction, $A_{d}=A_{z} / 2$ is given by [14]

$$
\mathrm{A}_{\mathrm{d}}=\frac{\mu_{0}}{4 \pi} \frac{\mathrm{g} \mu_{\mathrm{B}} \mathrm{g}_{\mathrm{N}} \mu_{\mathrm{N}}}{\mathrm{R}^{3}}
$$

with $\mu_{0}$ the vacuum magnetic permeability and $\mathrm{R}$ the distance between the center of paramagnetic electron density and the interacting nucleus. For the proton HF tensor of AAEPR I in Table 2 one calculates $R=330 \mathrm{pm}$. The principal $A_{x}$ and $A_{z}$ directions are tilted away by $6^{\circ} \pm 3^{\circ}$ from $<110>$ and $<001>$ directions, respectively.

Selected experimental ${ }^{1} \mathrm{H}$ ENDOR spectra recorded on the AA-EPR II spectrum are shown in Fig. 6. In general, they have poorer resolution and it is more difficult to follow their angular dependence. In Fig. 6a three $0^{\circ}$ spectra recorded in the $1150-1250 \mathrm{mT}$ range are shown, the field area dominated by the x-component of the AA-EPR II spectrum (see Section 
3.1). The spectra at 1170 and $1250 \mathrm{mT}$ should only contain contributions of the x-component, whereas in the $1209 \mathrm{mT}$ spectrum, y and z components should also be present. In the latter spectrum, indeed, three pairs of lines characterized by different splittings can be attributed to ${ }^{1} \mathrm{H}$ interactions and only one of those pairs (splitting 1.8 MHz, negative interaction parameter) persists in the other spectra. Measurements at other EPR transitions, as e.g. shown in Fig. $6 b(-7 / 2 \rightarrow-5 / 2$ transition of y-component), show that the pair with the largest splitting ( $4 \mathrm{MHz}$ ) corresponds to the y-component and the coupling has a positive sign. The intermediate, z-component splitting is negative and close to that observed for the perpendicular components for AA-EPR I. The limited angular dependent data did not allow a fitting of the full HF tensor. The tensor listed in Table 2 was obtained by fitting simulated to experimental spectra under the constraint $A_{x}=A_{y}$. As can be evaluated in Figs. 6a and $b$, reasonable agreement with experiment is obtained for the simulations using this tensor. Obviously, the experimental errors on principal values and directions are larger than for the corresponding tensor of AA-EPR I. The simulation of the FF-ENDOR spectrum in this region in Fig. $3 b$ also supports the ${ }^{1} \mathrm{H}$ ENDOR analysis for AA-EPR II. Three spots in the experimental spectrum (Fig. 3a) are, however, not explained by the simulation. The field dependence of the frequency position of the two lines at higher frequencies (55.7 and 57.5 $\mathrm{MHz}$ ) suggest that they are related with ${ }^{1} \mathrm{H}$ and they approximately occur at positions for the ${ }^{1} \mathrm{H}$ interaction for the $\mathrm{x}$-orientation of the AA-EPR II defect within other $\mathrm{M}_{\mathrm{S}}$ multiplets. The third spot exhibits a much smaller field dependence. The origin of these transitions is as yet not entirely clear.

The resemblance between the two tensors in Table 2 is striking. This certainly holds true for the principal values, and in addition the two tensors have their main (dipolar) axis close to a $<100>$ direction, albeit with a considerably larger tilting angle $\left(15^{\circ} \pm 6^{\circ}\right)$ in the case of AA-EPR II. Hence, in the two centers the ${ }^{1} \mathrm{H}$ nuclei occupy very similar positions with respect to the $\mathrm{Eu}^{2+}$ ion.

\section{$3.3{ }^{79 / 81} \mathrm{Br}$ and ${ }^{133} \mathrm{Cs}$ interactions}

The ENDOR spectra also exhibit an intense transition at the ${ }^{133} \mathrm{Cs}(\mathrm{I}=7 / 2,100 \%)$ Larmor frequency, which for certain magnetic field positions shows a splitting of a couple of $100 \mathrm{kHz}$. The ${ }^{79} \mathrm{Br}(\mathrm{I}=3 / 2,50.69 \%)$ and ${ }^{81} \mathrm{Br}(\mathrm{I}=3 / 2,49.31 \%)$ Larmor frequencies are also marked with intense ENDOR transitions and in a range of about $15 \mathrm{MHz}$ around them ENDOR lines with much lower intensity are detected. Because the structure of these spectra is quite complex (additional quadrupole splitting, large number of lines) and because they could 
only be recorded in a very small angular range we could not extract HF and quadrupole data from these measurements.

\section{Discussion}

\subsection{Similarities between the centers}

From our previous EPR study [6], and especially from the temperature dependence of the spectra, it is clear that AA-EPR I and II are very similar and also very closely related to the RT variant AA-EPR. Moreover, the present ENDOR results point to a very similar position for a $\mathrm{H}$-containing impurity near $\mathrm{Eu}^{2+}$ for AA-EPR I and II. These findings strongly indicate that the defects producing the AA-EPR type spectra have a common core. The similarity between the defects is, however, not entirely reflected in their ZFS parameters, as determined in our previous studies and shown in Table 1 . Indeed, the signs of $\mathrm{B}_{2}^{0}$ and $\mathrm{B}_{4}^{0}$ for AA-EPR II appear to be deviant. It should be noted, though, that the ZFS parameters are

given in their standard representation, taking the axes frame such that $\left|D_{x}\right|<\left|D_{y}\right|<\left|D_{z}\right|$ or $0<\mathrm{B}_{2}^{2} / \mathrm{B}_{2}^{0}<1$. In these axes systems, the principal ${ }^{1} \mathrm{H}$ HF tensor axis is practically collinear with the AA-EPR I z-axis, whereas for AA-EPR II this direction corresponds to the y-axis. For better comparison it might thus be advantageous to express the parameters of AA-EPR II in a frame where the $\mathrm{z}$ and $\mathrm{y}$-axes are interchanged. The result of such recalculation is also shown in Table 1. Indeed, the $\mathrm{B}_{4}^{0}$ parameter for AA-EPR II now also is negative and within experimental error has the same value as for AA-EPR and AA-EPR I. Also the negative $\mathrm{B}_{2}^{0}$ and positive $\mathrm{B}_{4}^{4}$ are now in line with the corresponding values for the other centers.

\subsection{Identification of the nearby impurity}

We previously [4-6] pointed to the fundamental difference between the AA-EPR type centers and the $\mathrm{Eu}^{2+}$-related centers produced in CsBr single crystals and NIPs after heating to $500-600^{\circ} \mathrm{C}$, whose EPR spectra rapidly decay at RT due to aggregation and which are presumably (as the similar centers in rock salt type alkali halides) associated with a cation vacancy. Hence, we proposed that a nearby impurity, related to the specific growth conditions, prevents the $\mathrm{Eu}^{2+}$ ions in NIPs from aggregating. Post annealing the plates was shown to have a positive effect on the PSL sensitivity and AA-EPR intensity of the plates and Weidner et al. [15] studied the effect of various annealing atmospheres. They demonstrated that the humidity of the atmosphere $\left(\mathrm{H}_{2} \mathrm{O}\right.$ partial pressure $)$ played a key role. Hence, $\mathrm{H}_{2} \mathrm{O}$ is a 
very plausible candidate as nearby impurity. In this subsection, we show that the ENDOR results are in agreement with such identification.

Figure 7 shows the geometry of a fictive $\mathrm{Eu}^{2+}-\mathrm{OH}_{2}$ molecule in a touching sphere representation. Using the known geometry of the water molecule $(\mathrm{O}-\mathrm{H}$ distance $95 \mathrm{pm}, \mathrm{H}-$ $\mathrm{O}-\mathrm{H}$ angle $\left.104.5^{\circ}\right)$ and average values of ionic radii for $\mathrm{O}^{2-}(140 \mathrm{pm})$ and $\mathrm{Eu}^{2+}(117 \mathrm{pm})$, one finds as $\mathrm{Eu}-\mathrm{H}$ distance $325 \mathrm{pm}$ with the $\mathrm{Eu}-\mathrm{H}$ axis tilted away by $13.7^{\circ}$ from the $\mathrm{Eu}-\mathrm{O}$ axis. The former result is in excellent agreement with the distance estimated from the ${ }^{1} \mathrm{H} \mathrm{HF}$ tensor in the point dipolar approximation (Section 3.2). The latter angle roughly corresponds to the estimated deviation of the ${ }^{1} \mathrm{H}$ principal $\mathrm{A}_{\mathrm{z}}$ direction from $\mathrm{a}<100>$ axis for AA-EPR II, but for AA-EPR I, where we consider this angle to be more reliably determined, the deviation is considerably larger. It should, however, be noted that in view of the limited angular range over which spectra have been measured and the needle structure of the specimens (not really single crystals) the accuracy of the experimentally determined tilting angle is limited. In addition, the presence of $\mathrm{Eu}^{2+}$ might also influence the electron density distribution around the $\mathrm{O}^{2-}$ ion and hence affect the $\mathrm{H}-\mathrm{O}-\mathrm{H}$ angle, having only a small effect on the $\mathrm{Eu}-\mathrm{H}$ distance but with a considerable effect on the O-Eu-H angle. And finally, if the HF tensor is not entirely due to dipolar interaction but also bears a small positive contribution through covalency effects, the $\mathrm{Eu}-\mathrm{H}$ distance might actually be larger than calculated from Eq. (4) and the O-Eu-H angle is also expected to be smaller. Although there is no direct evidence from ENDOR for the presence and/or position of oxygen in the AA-EPR type structures, the rudimentary calculations above provide a confirmation for the suspected association of $\mathrm{H}_{2} \mathrm{O}$ to $\mathrm{Eu}^{2+}$. Extended X-ray Absorption Fine Structure experiments may be considered to provide additional proof, although needles with higher dopant concentration would probably be needed for such measurements. In addition, our hypothesis that the $\mathrm{Eu}^{2+}$ ion has $\mathrm{a}_{2} \mathrm{O}$ ligand, does not contradict the interpretation of Hesse et al. [16] which proposed $\mathrm{Eu}^{2+}-\mathrm{O}^{2-}$ dipoles to be responsible for the enhanced PSL activity in annealed NIPs.

In principle, our ENDOR results cannot exclude the possibility that the nearby impurity be $\mathrm{OH}^{-}$instead of $\mathrm{H}_{2} \mathrm{O}$. However, it is then not obvious to explain the tilting of the ${ }^{1} \mathrm{H}$ HF tensors, unambiguously established by observing the inequivalence of ${ }^{1} \mathrm{H}$ nuclei when the magnetic field was rotated away from the needle $<001>$ axis. Moreover, as it is isovalent with $\mathrm{Br}^{-}$, one would expect $\mathrm{OH}^{-}$to occupy an anion position in the lattice, which is difficult to bring in accordance with the dipolar axis of the HF tensor being close to an $<001>$ axis. For these reasons we consider $\mathrm{OH}^{-}$as perturbing nearby impurity less probable. In principle careful infrared absorption measurements might also help in distinguishing between $\mathrm{H}_{2} \mathrm{O}$ or 
$\mathrm{OH}^{-}$as ligand for the $\mathrm{Eu}^{2+}$ ion. Such measurements, both on samples annealed in $\mathrm{H}_{2} \mathrm{O}$ and in $\mathrm{D}_{2} \mathrm{O}$ atmosphere, have been attempted but failed to provide an answer, mainly because an intense absorption band of surface water on the needles, which proved very difficult to remove, obscured the spectral range of interest.

\subsection{Model for the AA-EPR type centers}

Based on literature data for di- and trivalent transition metal impurities in $\mathrm{CsBr}$ [1722] we put forward a model in Ref. [6] which in principle could explain the observed temperature dependence of the spectra. It is depicted for AA-EPR I and II in Figs. 8a and b, respectively. In these models, the $\mathrm{Eu}^{2+}$ ion takes an interstitial position in (or near) the center of four $\mathrm{Br}^{-}$ions. The extra charge $+2 \mathrm{e}$ is compensated by two cation vacancies along the $<100>$ axis perpendicular to the $\left(\mathrm{EuBr}_{4}\right)^{2-}$ plane of the defect, and the $\mathrm{Eu}^{2+}$ ion has two $\mathrm{H}_{2} \mathrm{O}$ molecules on the same axis as $5^{\text {th }}$ and $6^{\text {th }}$ ligand. At RT, the $\mathrm{H}_{2} \mathrm{O}$ molecules would be able to freely rotate around the $\mathrm{H}_{2} \mathrm{O}-\mathrm{Eu}-\mathrm{OH}_{2}$ axis and the axial AA-EPR defect would be observed. At low temperatures this motion would freeze out, leaving the $\mathrm{H}_{2} \mathrm{O}$ molecules in $\{100\}$ planes, perpendicular (AA-EPR I) or parallel (AA-EPR II) to one another. The fact that $\mathrm{Eu}^{2+}$ would have $\mathrm{H}_{2} \mathrm{O}$ ligands seems indeed supported by the ENDOR results (see Section 4.2), but still, on quantitative grounds, one may argue that this model for the AA-EPR type defects seems little plausible. Indeed, the O-Eu-O core is expected to impose the main axial symmetry, whereas the four protons should only produce a small perturbation. It is hard to imagine that changing the position of these four protons would change the symmetry from axial to nearly extremely rhombic, producing a contribution to the ZFS larger than half of that due to the O-Eu-O core (cfr. change of the principal $D_{z}$ direction from AA-EPR I to AA-EPR II in the standard representation). Moreover it is difficult to explain why the AA-EPR II center would have a higher abundance, since there seems to be no obvious reason for its particular arrangement of protons to be more stable.

For these reasons, we consider models in which a basic structure, consisting of a $\mathrm{Eu}^{2+}$ ion surrounded by one or several $\mathrm{H}_{2} \mathrm{O}$ molecules, is perturbed by an intrinsic lattice defect (e.g. a vacancy) as better suited to explain the observed effects. Furthermore, it is easier to device models rendering the orthorhombic-II symmetry (with $<100>$ principal axes) of AAEPR II starting from a $\mathrm{Eu}^{2+}$ ion at (or near) the substitutional $\mathrm{Cs}^{+}$position. Probably the simplest models one can construct in this way, are shown in Figs. $8 \mathrm{c}$ and d. They consist of a substitutional $\mathrm{Eu}^{2+}$ ion, charge compensated by a $\mathrm{H}_{2} \mathrm{O}$ molecule in one of the nearest $\mathrm{Cs}^{+}$ cages, defining the defects' $\mathrm{z}$ (or y) axis. Although this defect is electrically neutral, the 
presence of a nearby cation vacancy may still be favorable because it can accommodate the mechanical stress induced in the lattice by exchanging a $\mathrm{Cs}^{+}$ion for a smaller $\mathrm{Eu}^{2+}$ and by introducing water. From the five remaining nearest-neighbour $\mathrm{Cs}^{+}$positions next to $\mathrm{Eu}^{2+}$, only one produces a defect with axial symmetry (AA-EPR I, Fig. 8c), whereas the other four are equivalent and in principle lead to monoclinic-II symmetry (one principal axis along a $<100>$ direction). However, one may expect one other principal defect axis to be close to the Eu- $\mathrm{OH}_{2}$ axis, and the symmetry observed for the center may be (close to) orthorhombic-II. If these two inequivalent vacancy configurations are close in energy and if at sufficiently high temperatures the vacancy can hop between these positions, effectively an axial spectrum would be observed. The higher intensity for the AA-EPR II spectrum at low temperatures is then also explained in a natural way by the higher number of equivalent positions for this vacancy configuration. As both an $\mathrm{H}_{2} \mathrm{O}$ molecule "replacing" $\mathrm{Cs}^{+}$and a $\mathrm{Cs}^{+}$vacancy represent an effective charge -e in the lattice, their contributions to the ZFS parameters (effect on excited states level splitting) might be of the same order of magnitude, which makes the transition from axial to nearly extremely rhombic when going from model $\mathrm{c}$ to $\mathrm{d}$ actually quite plausible. Finally, it should be noted that in model d the two protons are possibly not exactly equivalent, which might be a reason for the poorer quality of the spectra and may also explain the worse result of fitting (in which equivalent ${ }^{1} \mathrm{H}$ nuclei were assumed).

If the $\mathrm{Eu}^{2+}$ ion and the $\mathrm{H}_{2} \mathrm{O}$ molecule would both be exactly in substitutional positions, the Eu-H distance would be $490 \mathrm{pm}$, considerably larger than that estimated from the ${ }^{1} \mathrm{H} H F$ coupling strength. As mentioned in Section 4.2 the Eu-H distance may be larger than the calculated $320 \mathrm{pm}$ if covalency effects play a role. Moreover, it may be expected that $\mathrm{Eu}^{2+}$ and the $\mathrm{O}^{(2-)}-\mathrm{H}_{2}{ }^{(2+)}$ dipole relax towards one another. The attractive force between these two impurities may in addition prevent the $\mathrm{Eu}^{2+}$ ions from aggregating.

One may invoke the charge imbalance (overall charge -e) as an argument against these models. It should, however, be noted that CsBr:Eu NIPs contain Br vacancies, which are the primary (photostimulable) electron traps, and may act as non-local charge compensators. The presence of such negatively charged defects might indeed enhance the concentration of $\mathrm{Br}$ vacancies and/or act as hole traps, two effects which enhance the PSL sensitivity. Hence, the charge imbalance might actually (in part) explain the observed correlation between EPR intensity of the AA-EPR centers and the PSL sensitivity of the plate [5].

Although the models in Fig. $8 \mathrm{c}$ and $\mathrm{d}$ are capable of explaining a large number of experimental observations, it should be noted that the ENDOR experiments do not provide final proof for them and we do not want to claim that they are the only models in agreement 
with all EPR and ENDOR results. Hyperfine interactions with surrounding ${ }^{133} \mathrm{Cs}$ and ${ }^{79 / 81} \mathrm{Br}$ nuclei can in principle provide additional information. For the $\mathrm{Cu}^{2+}$ centers in $\mathrm{NH}_{4} \mathrm{Cl}$ and $\mathrm{NH}_{4} \mathrm{Br}$, e.g., which have the same crystal structure as $\mathrm{CsBr}$ at low temperature, the observation of strong $\mathrm{HF}$ interactions with ${ }^{35 / 37} \mathrm{Cl}\left(\mathrm{A}_{\text {iso }}=15-20 \mathrm{MHz}\right.$ [17] $)$ and ${ }^{79 / 81} \mathrm{Br}\left(\mathrm{A}_{\text {iso }}\right.$ $25 \mathrm{MHz}$ [22]) nuclei, respectively, in the $g_{x}-g_{y}$ plane of the defects provided proof for the interstitial position of the $\mathrm{Cu}^{2+}$ ions. We did not observe ${ }^{79 / 81} \mathrm{Br}$ interactions of this size in the ENDOR spectra, but in principle the residual line width of the EPR spectra could accommodate such interactions. Also the smaller interactions mentioned in Section 3.3 may bear key information on the structural models, but the limited number of spectra we could record for them did not allow for a full analysis.

Moreover, the models here proposed do not explain how the AA-EPR centers are incorporated in the needles during growth or how their concentration would be increased during post annealing. Possibly, they are generated near the surface, where a higher concentration of (aggregated) $\mathrm{Eu}^{2+}$ ions may be present, after which they might migrate into the bulk of the needles. Recent time-of-flight secondary ion mass spectroscopy measurements, indeed, demonstrate a higher Eu concentration near the needle surface [23]. The present models also do not provide a direct explanation for the recent X-ray absorption near-edge structure results, indicating a partial oxidation $\left(\mathrm{Eu}^{2+}\right.$ to $\left.\mathrm{Eu}^{3+}\right)$ by a few percent in the needles by the same annealing procedure which enhances the concentration of AA-EPR type centers [24]. And finally, the complexity of the models, involving, next to the $\mathrm{Eu}^{2+}$ ion, both an impurity and a vacancy, also raises the question why other combinations would not be produced, or at least are not observed in EPR and ENDOR experiments. This may indicate that the defect structures proposed in Fig. 8c and d are energetically very favorable, a hypothesis which may be further explored by first principles calculations.

\section{Conclusions}

Studying the field-dependence of the ENDOR spectrum for the AA-EPR I and EPREPR II components of the low temperature EPR spectrum of CsBr:Eu NIPs, we were able to attribute the most intense transitions in the $40-150 \mathrm{MHz}$ region to ${ }^{151 / 153} \mathrm{Eu}$ and ${ }^{1} \mathrm{H}$ nuclei. A thorough analysis for the ${ }^{1} \mathrm{H}$ interactions unambiguously demonstrates that in the corresponding defects, the $\mathrm{Eu}^{2+}$ ion is associated with a H-containing impurity. In both centers the $\mathrm{Eu}^{2+}-\mathrm{H}$ interconnection line is found close to a $<100>$ direction. Moreover, the ${ }^{1} \mathrm{H} \mathrm{HF}$ coupling strength suggests that the proton belongs to a $\mathrm{H}_{2} \mathrm{O}$ ligand of the $\mathrm{Eu}^{2+}$ ion. Expressing the ZFS parameters for the three AA-EPR type defects (including the RT variant) in a 
common axis system, taking the Eu- $\mathrm{OH}_{2}$ axis as z-axis, reveals striking similarities between them. We therefore assume they have a common core, differing only in the position of an additional perturbing lattice defect. In the models here proposed, we assume the $\mathrm{Eu}^{2+}$ ion and $\mathrm{H}_{2} \mathrm{O}$ molecule to (approximately) occupy nearest neighbour $\mathrm{Cs}^{+}$positions and to exhibit an important relaxation towards one another. The perturbing defect is proposed to be a cation vacancy in one of the five remaining nearest neighbour positions to the $\mathrm{Eu}^{2+}$ ion. The position of the vacancy, either along the $\mathrm{Eu}-\mathrm{OH}_{2}$ axis or in one of the four equivalent positions in the plane perpendicular to it, explains the difference between the AA-EPR I and AA-EPR II defects at low temperature, while hopping of the vacancy between these positions at elevated temperatures produces the axial AA-EPR spectrum. Quantum chemical calculations are necessary in order to verify the stability of these models for the AA-EPR type defects, which apparently play a key role in the PSL process in CsBr:Eu NIPs, as well as for revealing their formation mechanisms.

\section{Acknowledgements}

The authors wish to acknowledge financial support from the Flemish Research Foundation (FWO-Vlaanderen) through research grant $n^{\circ}$ G.0116.06. One of the authors acknowledges a Postdoctoral Fellowship with the same institution.

\section{References}

1. J.A. Rowlands, Phys. Med. Biol. 47, R123 (2002).

2. P. Leblans, L. Struye and P. Willems, Phys. Med. Imaging Proc. SPIE 4320, 59 (2001).

3. J. M. Spaeth, Rad. Meas. 33, 527 (2001).

4. F. Loncke, H. Vrielinck, P. Matthys, F. Callens, J.-P. Tahon and P. Leblans, Spectrochim. Acta A 69, 1322 (2008).

5. F. Loncke, H. Vrielinck, P. Matthys, F. Callens, J.-P. Tahon and P. Leblans, Appl. Phys. Lett. 92, article no. 204102 (2008).

6. F. Loncke, H. Vrielinck, P. Matthys, F. Callens, J.-P. Tahon and P. Leblans, Phys. Rev. $B$ 79, article no. 174102 (2009).

7. S. Schweizer, U. Rogulis, S. Assman and J.-M. Spaeth, Radiat. Meas. 33, 483 (2001).

8. V. P. Savelev, V. P. Avdonin, L. D. Dugarova, A. P. Nedashkovskii, and B. T. Planchenov, Sov. Phys. Solid State 16, 700 (1974).

9. J. O. Rubio, J. Phys. Chem. Solids 52, 101 (1991). 
10. S. Stoll and A. Schweiger, J. Magn. Reson. 178, 42 (2006).

11. C. Rudowicz, J. Phys. Condens. Matter 12, L417 (2000).

12. F. Loncke, H. De Cooman, N. M. Khaidukov, H. Vrielinck, E. Goovaerts, P. Matthys and F. Callens, Phys. Chem. Chem. Phys. 9, 5320 (2007).

13. H. Vrielinck, H. De Cooman, Y. Karakirova, N. D. Yordanov, and F. Callens, Radiat. Res. 172, 226 (2009).

14. J.-M. Spaeth, J. R. Niklas and R. H. Bartram, Structural analysis of point defects in solids : an introduction to multiple magnetic resonance spectroscopy, Springer-Verlag, 1992.

15. M. Weidner, Einfluss der Teperbedingungen auf Lumineszenz- und Speicherzentren in CsBr: $E u^{2+}$ Nadelbildplatten, PhD. Thesis, University of Erlangen, Hut Verlag, 2009.

16. S. Hesse, J. Zimmerman, H. von Seggern, H. Eherenberg, and H. Fuess, Radiat. Meas. 42, 638 (2007).

17. F. Boettcher and J.-M. Spaeth, Phys. Status Solidi b 61, 465 (1974).

18. F. Boettcher and J.-M. Spaeth, Phys. Status Solidi b62, 65 (1974).

19. K. Watanabe and A. Hidetaro, J. Phys. Soc. Jpn. 38, 755 (1975).

20. F. S. Stibbe and N. J. Trappeniers, Phys. B\&C 95, 81 (1978).

21. T. Behner and G. Lehmann, J. Phys. Chem. Solids 48, 555 (1987).

22. W. Sano and E. Di Mauro, J. Phys. Chem. Solids 58, 391 (1997).

23. Agfa-HealthCare, private communication.

24. J.-P. Tahon, J. Lenaerts, L. Vincze, G. Silversmit and P. Leblans, European Patent Application EP 2067841 A1 (2009). 


\section{Figure Captions}

Figure 1 : Q-band EPR spectra (34 GHz) of CsBr:Eu NIPs (a) at room temperature, recorded with the magnetic field along the needle $<100>$ axis : the $\mathrm{z}$ (green) and $\mathrm{xy}$ (red) spectral components of the AA-EPR center are indicated in full arrows ; and (b) at $4 \mathrm{~K}$ : spectral components of AA-EPR I are indicated with full arrows (green - z/xy - red) and of AA-EPR II with dashed arrows/lines (cyan - z/purple - y/orange $-\mathrm{x}$ ).

Figure 2 : a) FF-ENDOR spectrum of CsBr:Eu NIPs recorded with the magnetic field along the needle $<100>$ axis at $3.5 \mathrm{~K}$ in the $1150-1250 \mathrm{mT}$ field range. b) Experimental EPR spectrum in this field range, and c) simulation of the spectral $\mathrm{x}$-component for the AA-EPR II center (parameters in Table 1). d) Horizontal section (indicated in a) with dash-dotted line) of the FF-ENDOR spectrum, corresponding to the normal ENDOR spectrum at $1200 \mathrm{mT}$. e) Simulation of the ${ }^{151 / 153}$ Eu FF-ENDOR spectrum for the AA-EPR II center using the HF and quadrupole parameters in the text (Section 3.1). Dashed ellipses in a) and e) indicate the $\mathrm{x}$ component ${ }^{151} \mathrm{Eu}$ transitions within the $\mathrm{M}_{\mathrm{S}}=-3 / 2$ multiplet. Black and white arrows correspond to ${ }^{153} \mathrm{Eu}$ transitions (x-component) within the $\mathrm{M}_{\mathrm{S}}=-5 / 2$ and $-7 / 2$ multiplets, respectively.

Figure 3 : Detail of the FF-ENDOR spectrum in Fig. 2 in the $40-60 \mathrm{MHz}$ frequency range, near the ${ }^{1} \mathrm{H}$ larmor frequency, indicated with a full white line. The parallel dashed white lines, serving as guides for the eye, show that certain ENDOR transitions exhibit the same field dependence as the ${ }^{1} \mathrm{H}$ larmor frequency. a) Experimental spectrum, b) simulation using the ${ }^{1} \mathrm{H}$ HF tensor for AA-EPR II in Table 2. Intense transitions not accounted for by the simulation are indicated in a) by full black ellipses.

Figure 4 : Selected experimental (black) and simulated (red) ${ }^{1} \mathrm{H}$ ENDOR spectra recorded in a limited angular range around the $<100>$ needle axis $\left(0^{\circ}\right)$ for AA-EPR I. The magnetic field positions at which the spectra were recorded are indicated on the right of each spectrum, the defect orientations and $\mathrm{M}_{\mathrm{S}}$ initial and final states of the EPR transition on which ENDOR is recorded for part a) and b) are also indicated.

Figure 5 : Simulated full angular dependence of the ${ }^{1} \mathrm{H}$ ENDOR transitions of AA-EPR I when following the $\mathrm{M}_{\mathrm{S}}=-5 / 2 \rightarrow-3 / 2$ transition in the $\mathrm{z} \rightarrow \mathrm{x}, \mathrm{y}$ and the $\mathrm{x}, \mathrm{y} \rightarrow \mathrm{y}, \mathrm{x}$ planes using the HF tensor in Table 2. Due to the tilting of the principal $A_{z}$ and $A_{x}$ axes in the $\{101\}$ plane 
the transitions within the $M_{S}=-5 / 2$ and $-3 / 2$ multiplets both split in two components when the magnetic field is rotated away from a $<100>$ axis. For comparison the ${ }^{1} \mathrm{H}$ Larmor frequency is indicated as a black full line. Experimental data points, corrected for the difference in magnetic field between experimental and simulation conditions, are indicated with diamonds.

Figure 6 : Selected experimental (black) and simulated (green $-\mathrm{x}$; red $-\mathrm{y}$; blue $-\mathrm{z}$ ) ${ }^{1} \mathrm{H}$ ENDOR spectra recorded for AA-EPR II a) with the magnetic field along the needle $<100>$ axis at three magnetic field positions in the 1150 - $1250 \mathrm{mT}$ range and b) limited angular dependence on the $\mathrm{y} \rightarrow \mathrm{x}, \mathrm{z}$ component of the $\mathrm{M}_{\mathrm{S}}=-7 / 2 \rightarrow-5 / 2$ EPR transition. The transitions indicated with an asterisk in the bottom spectrum of a) correspond to the three unexplained spots (circles) in the FF-ENDOR spectrum of Fig. 3a.

Figure $7: \mathrm{Eu}^{2+}-\mathrm{OH}_{2}$ entity in a touching ionic spheres representation. If the Eu-O axis is assumed to be parallel to a crystal $<100>$ axis, the principal $\mathrm{A}_{\mathrm{z}}$ direction of the ${ }^{1} \mathrm{H}$ HF tensors (Table 2) is expected to be oriented (approximately) along the $\mathrm{Eu}-\mathrm{H}$ interconnection line.

Figure 8: Models for the AA-EPR I (a and c) and AA-EPR II (b and d) centers. Models a and $b$, in which $\mathrm{Eu}^{2+}$ occupies an interstitial position, were suggested in our previous paper [6] to be able to provide a qualitative explanation for the temperature dependence of the spectra. Model a has tetragonal $\left(D_{2 d}\right)$ symmetry and b orthorhombic $\left(D_{2 h}\right)$. When the $\mathrm{H}_{2} \mathrm{O}$ molecules are allowed to rotate at elevated temperatures, effective tetragonal symmetry $\left(\mathrm{D}_{4 \mathrm{~h}}\right)$ is observed. Models $\mathrm{c}$ and $\mathrm{d}$ with the $\mathrm{Eu}^{2+}$ on a substitutional position are currently proposed, based on the ENDOR results of this paper. Model $c$ has tetragonal $\left(\mathrm{C}_{4 \mathrm{v}}\right)$ symmetry and $\mathrm{d}$ is approximately orthorhombic (in principle it only has the zx mirror plane). When the cation vacancy is allowed to hop between the five remaining cation positions nearest to the $\mathrm{Eu}^{2+}$ at high temperature, tetragonal symmetry $\left(\mathrm{C}_{4 \mathrm{v}}\right)$ is expected. 


\section{Tables}

Table 1. $g$ and ZFS parameters measured at $4 \mathrm{~K}$ of AA-EPR, AA-EPR I and AA-EPR II (left column) as determined in [6], defining the xyz axes frame of the defects (see text). The left column values for AA-EPR II are given in the standard representation [11] with $0<\mathrm{B}_{2}^{2} / \mathrm{B}_{2}^{0}<1$, the right column values are obtained by fitting the resonance fields calculated with the left column values exchanging the defect's y and z-axes. Numbers in subscript indicate estimates of experimental error.

\begin{tabular}{|c|c|c|}
\hline \multicolumn{3}{|c|}{ AA-EPR } \\
\hline $\mathrm{g}$ & \multicolumn{2}{|c|}{$1.991_{5}$} \\
\hline $\mathrm{B}_{2}^{0}[\mathrm{MHz}]$ & \multicolumn{2}{|c|}{$-755_{11}$} \\
\hline $\mathrm{B}_{4}^{0}[\mathrm{MHz}]$ & \multicolumn{2}{|c|}{$-0.27_{18}$} \\
\hline $\mathrm{B}_{4}^{4}[\mathrm{MHz}]$ & \multicolumn{2}{|c|}{$3.47_{99}$} \\
\hline \multicolumn{3}{|c|}{ AA-EPR I } \\
\hline $\mathrm{g}$ & \multicolumn{2}{|c|}{$1.993_{5}$} \\
\hline $\mathrm{B}_{2}^{0}[\mathrm{MHz}]$ & \multicolumn{2}{|c|}{$-964.6_{6.9}$} \\
\hline $\mathrm{B}_{4}^{0}[\mathrm{MHz}]$ & \multicolumn{2}{|c|}{$-0.27_{10}$} \\
\hline $\mathrm{B}_{4}^{4}[\mathrm{MHz}]$ & \multicolumn{2}{|c|}{$4.77_{65}$} \\
\hline \multicolumn{3}{|c|}{ AA-EPR II } \\
\hline $\mathrm{g}$ & $1.991_{4}$ & 1.991 \\
\hline $\mathrm{B}_{2}^{0}[\mathrm{MHz}]$ & $815.6_{9.1}$ & -797 \\
\hline $\mathrm{B}_{2}^{2}[\mathrm{MHz}]$ & $778_{21}$ & -834 \\
\hline $\mathrm{B}_{4}^{0}[\mathrm{MHz}]$ & $0.59_{14}$ & -0.27 \\
\hline $\mathrm{B}_{4}^{2}[\mathrm{MHz}]$ & $-1.7_{1.2}$ & 1.7 \\
\hline $\mathrm{B}_{4}^{4}[\mathrm{MHz}]$ & $-2.2_{1.2}$ & 3.8 \\
\hline
\end{tabular}


Table 2. Principal values and eigenvectors in the defect principal axes frame (determined by the ZFS Hamiltonian in its standard representation) of the ${ }^{1} \mathrm{H}$ hyperfine tensor for AA-EPR I and AA-EPR II. Numbers in subscript indicate estimates of experimental error. The $\mathrm{A}_{\mathrm{y}}$ axes are assumed to be directed exactly along a crystallographic direction. The principal directions corresponding to $A_{x}$ and $A_{z}$ tilted away by $6^{\circ} \pm 3^{\circ}$ from $<110>$ and $<001>$ for the AA-EPR I center and by $15^{\circ} \pm 6^{\circ}$ from $<100>$ directions for AA-EPR II.

\begin{tabular}{|ccccc||}
\hline & $\begin{array}{c}\text { Principal } \\
\text { value }\end{array}$ & $\mathrm{x}$ & Eigenvectors & $\mathrm{z}$ \\
$\boldsymbol{A} \boldsymbol{A}$ - $\boldsymbol{E} \boldsymbol{P} \boldsymbol{R} \boldsymbol{I}$ & & & & \\
$\mathrm{A}_{\mathrm{x}}$ & $-2.4_{1}$ & $0.703_{4}$ & $-0.703_{4}$ & $0.108_{37}$ \\
$\mathrm{~A}_{\mathrm{y}}$ & $-2.0_{1}$ & 0.707 & 0.707 & 0 \\
$\mathrm{~A}_{\mathrm{z}}$ & $4.7_{1}$ & $-0.076_{52}$ & $-0.076_{52}$ & $0.994_{6}$ \\
& & & & \\
$\boldsymbol{A} \boldsymbol{A}$ - $\boldsymbol{E} \boldsymbol{P} \boldsymbol{R} \boldsymbol{I I}$ & & & $0.260_{100}$ & 0 \\
$\mathrm{~A}_{\mathrm{x}}$ & $-2.4_{3}$ & $-0.966_{27}$ & 0 & 1 \\
$\mathrm{~A}_{\mathrm{y}}$ & $-2.4_{3}$ & 0 & $0.966_{27}$ & 0 \\
$\mathrm{~A}_{\mathrm{z}}$ & $4.6_{3}$ & $0.260_{100}$ & & \\
\hline
\end{tabular}




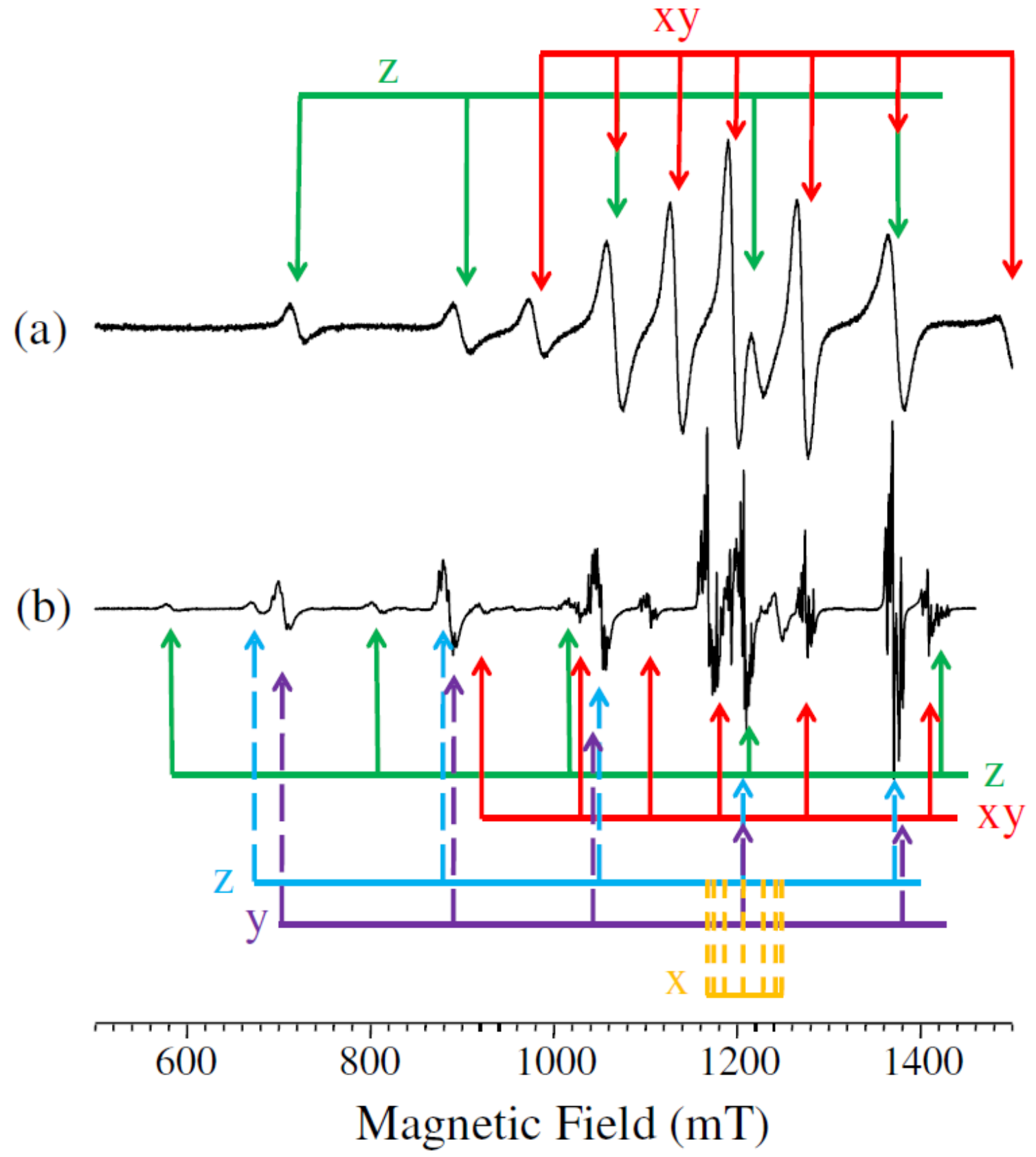

Figure 1 


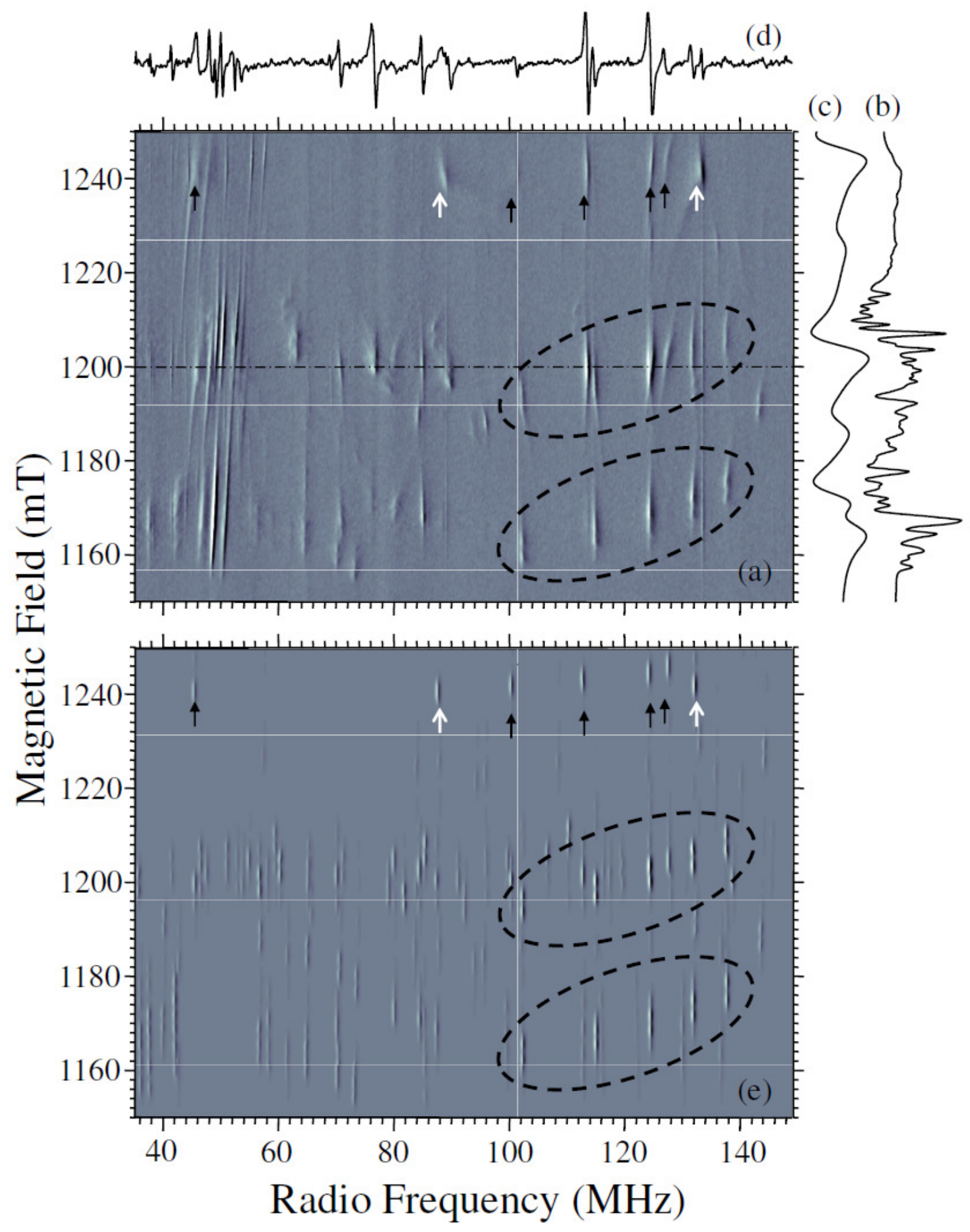

Figure 2 


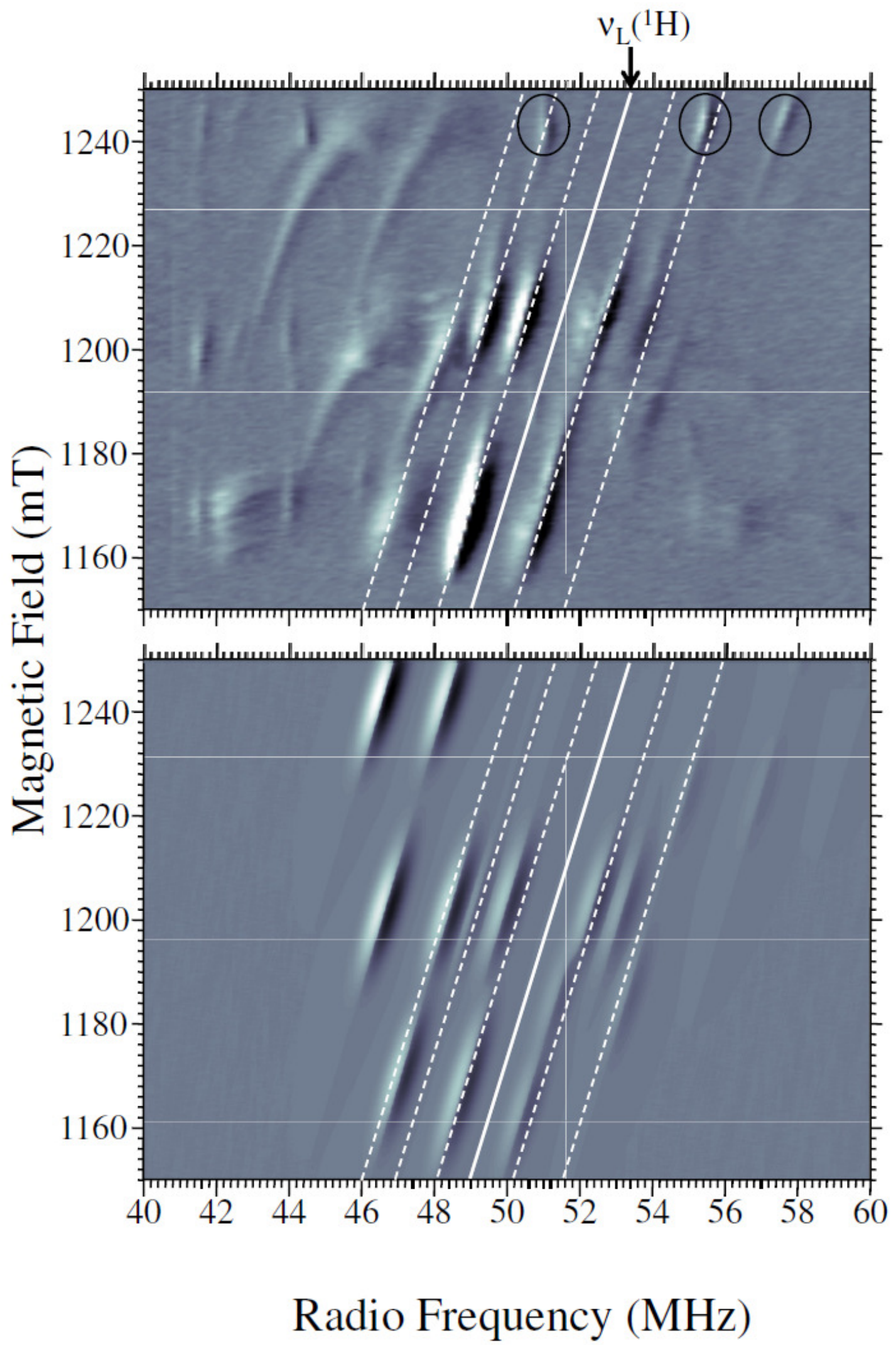

Figure 3 
(a)

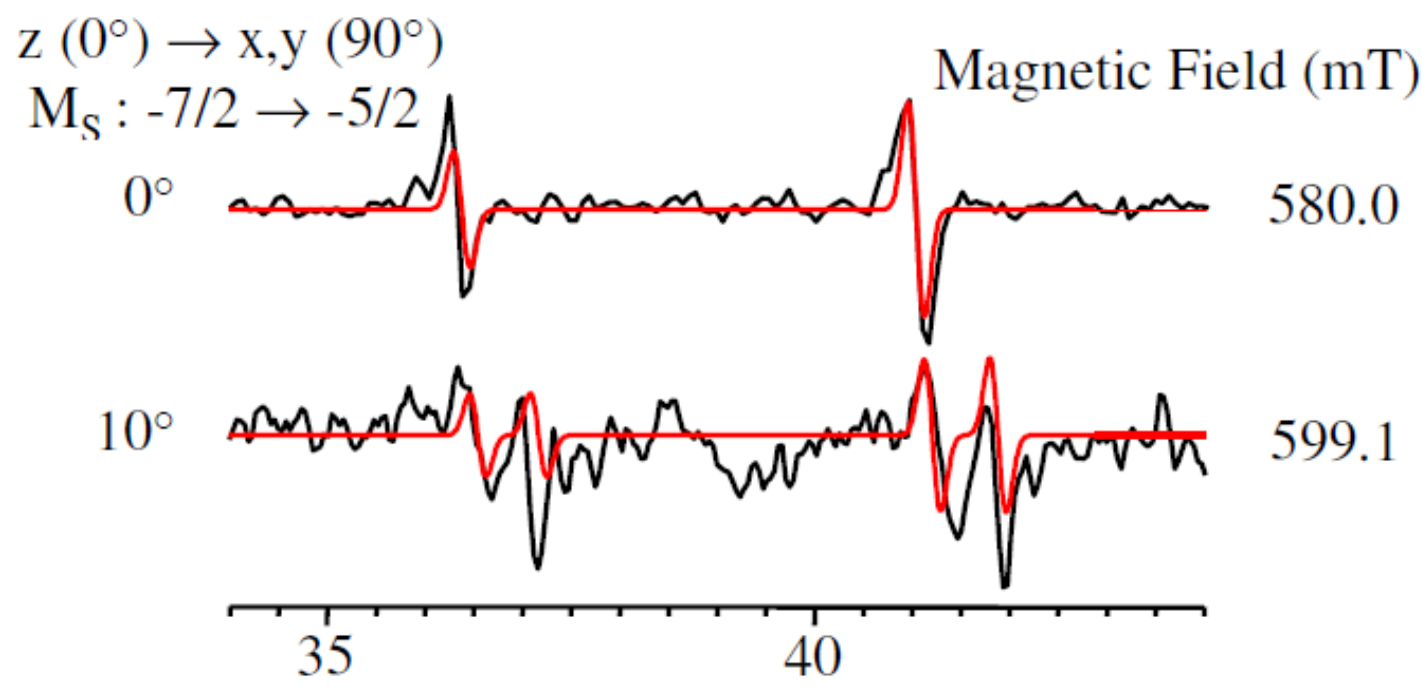

(b)

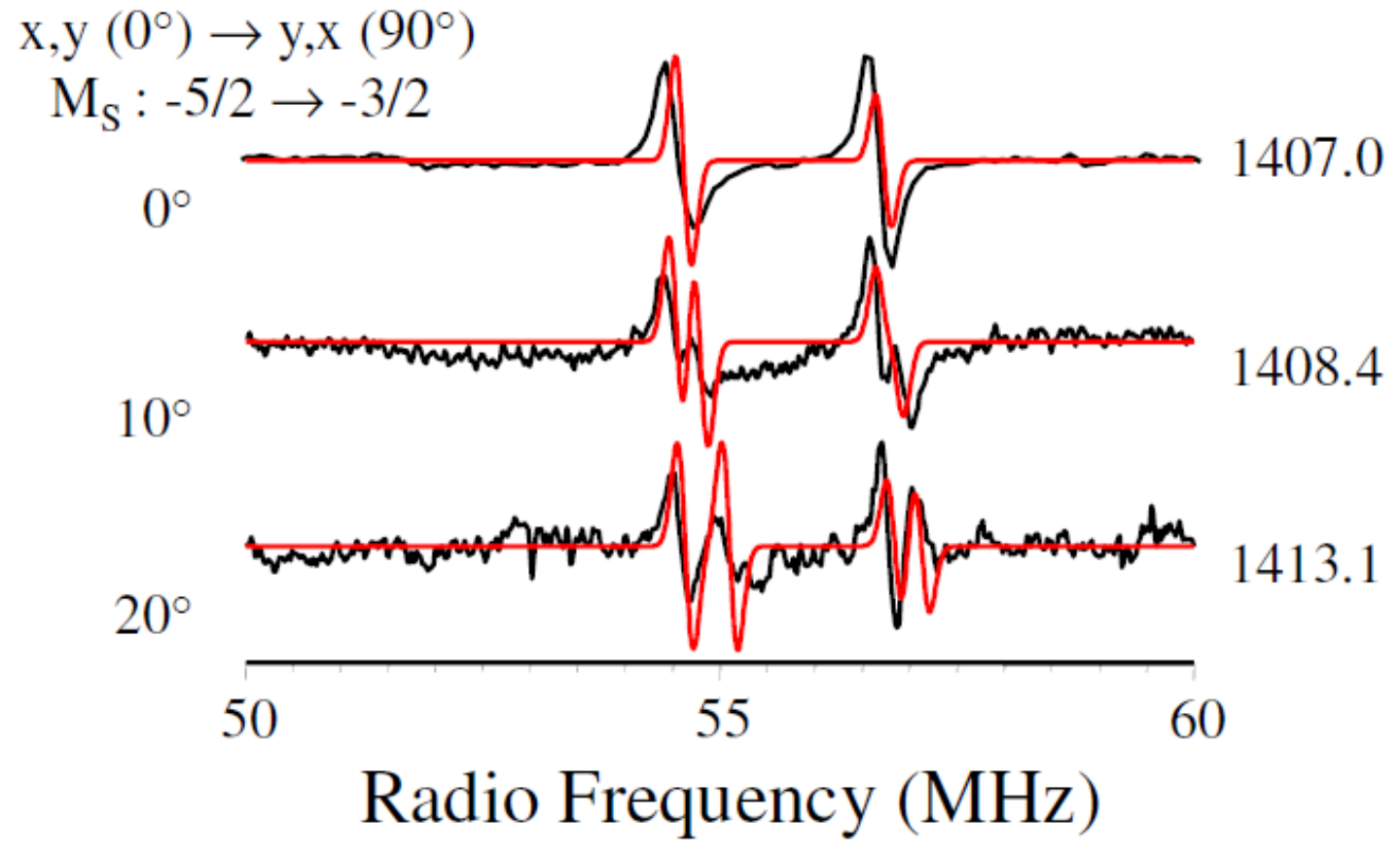

Figure 4 


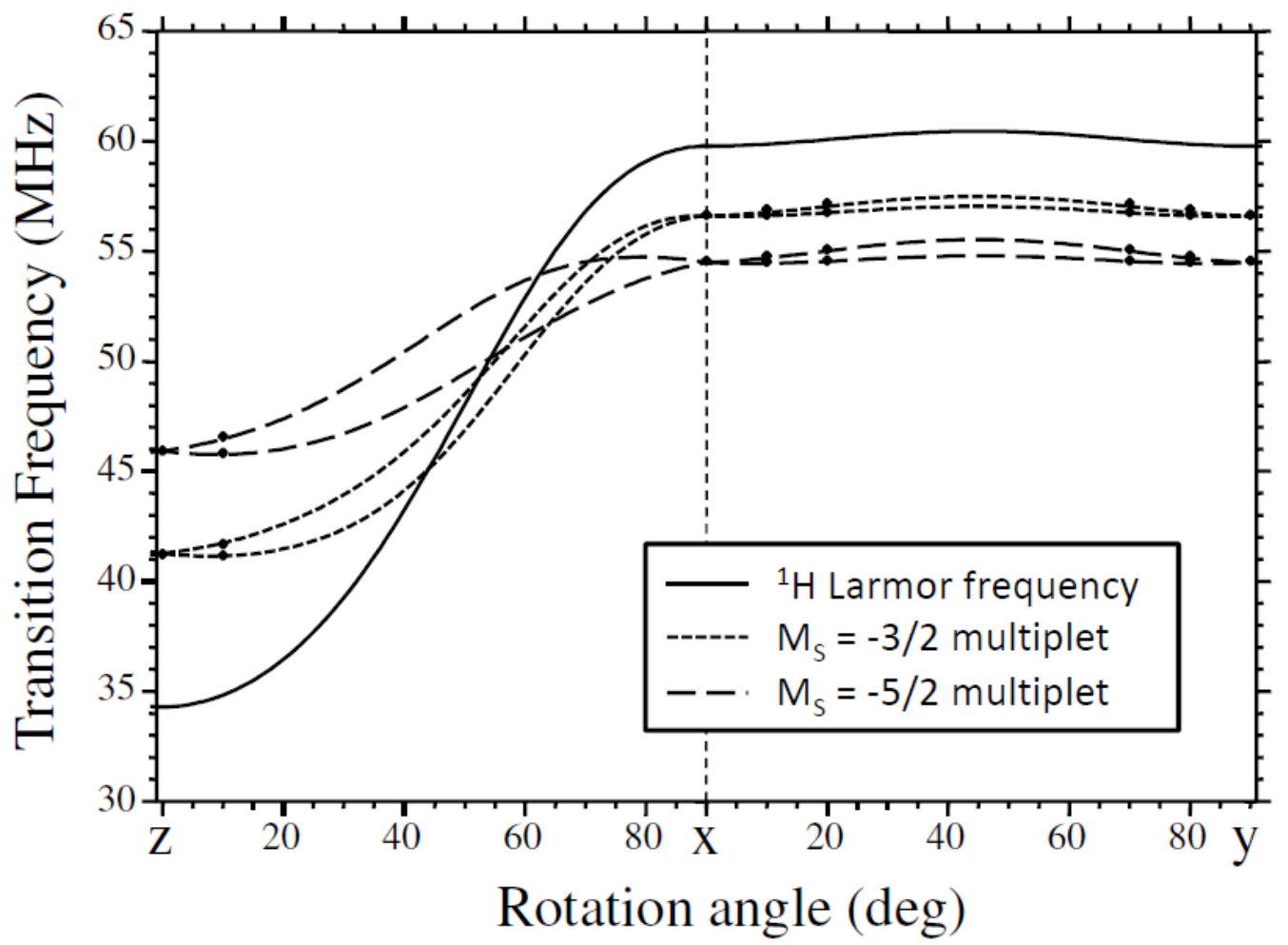

Figure 5 


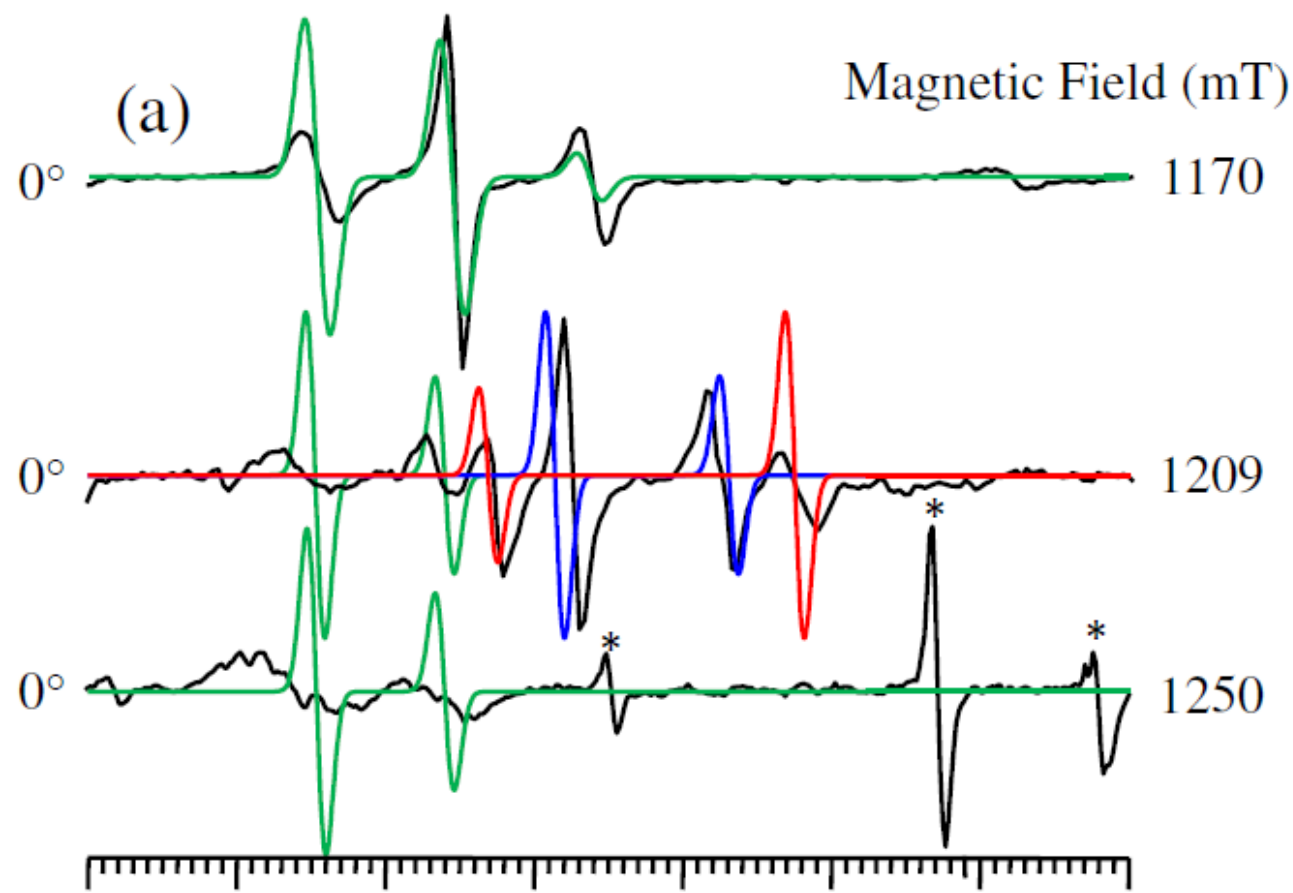

$\begin{array}{llllllll}44 & 46 & 48 & 50 & 52 & 54 & 56 & 58\end{array}$

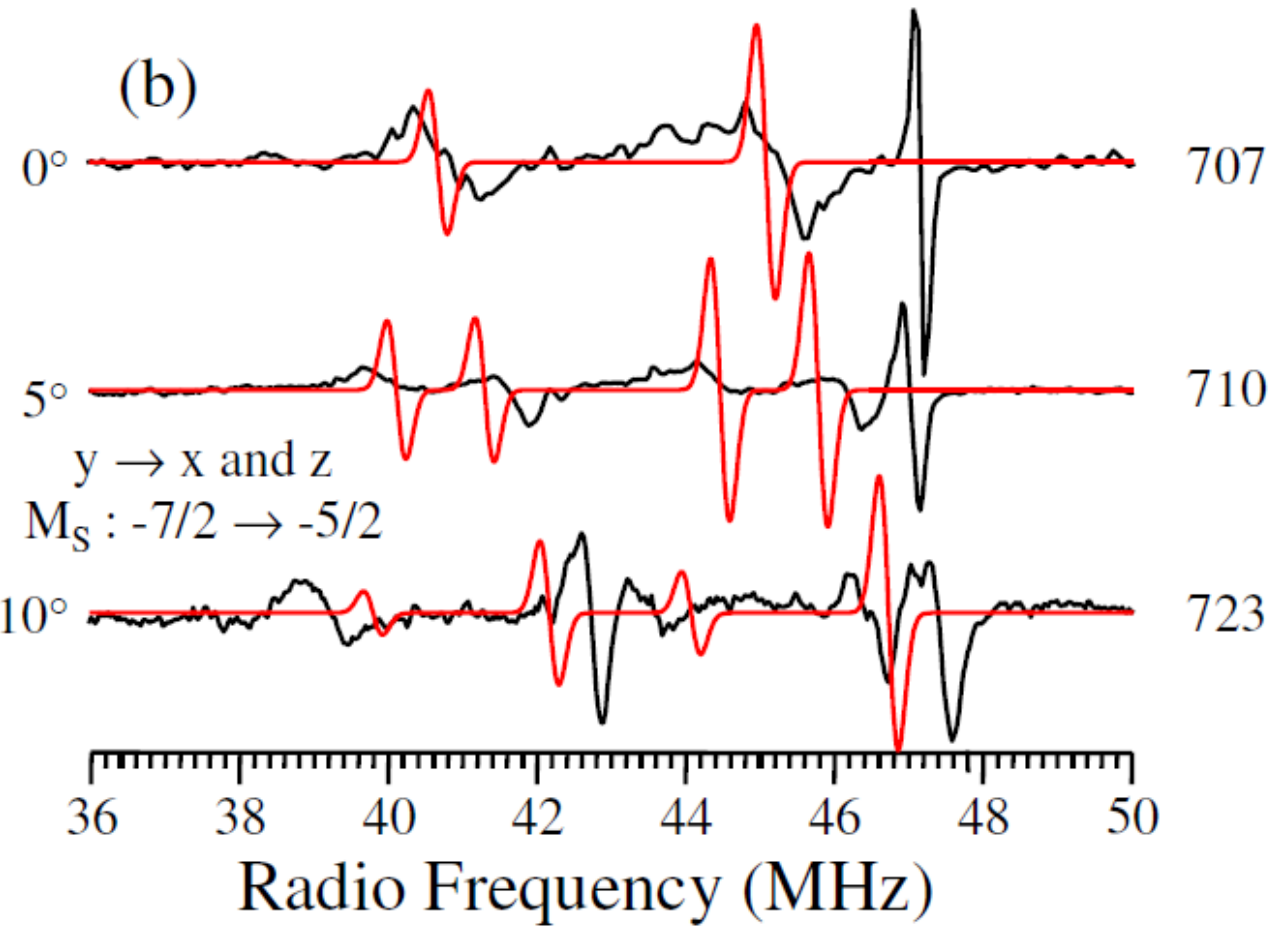

Figure 6 


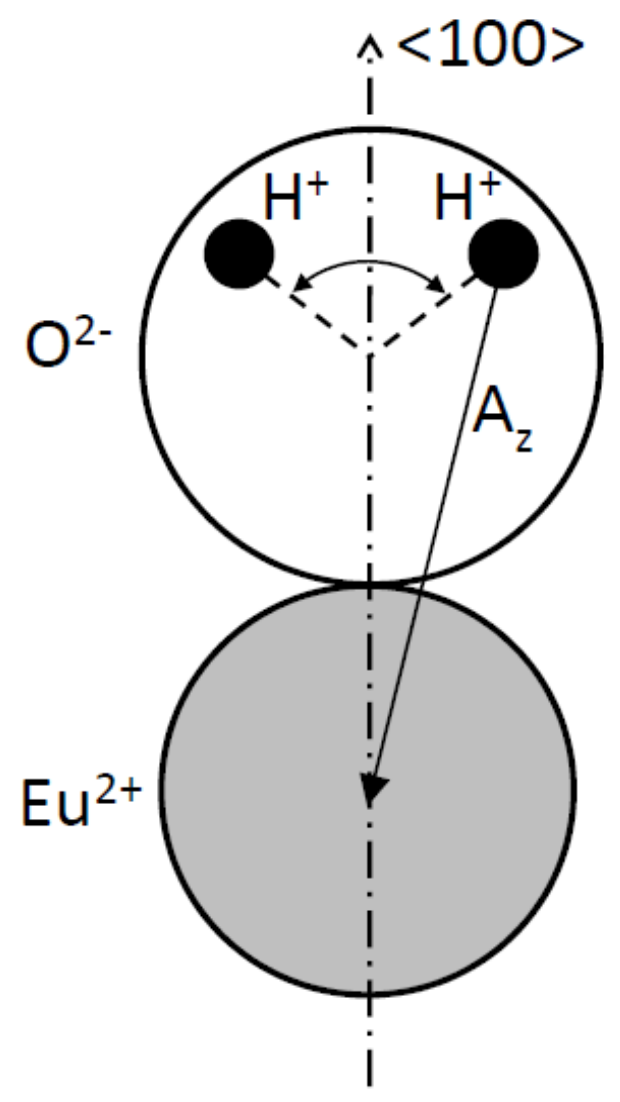

Figure 7 


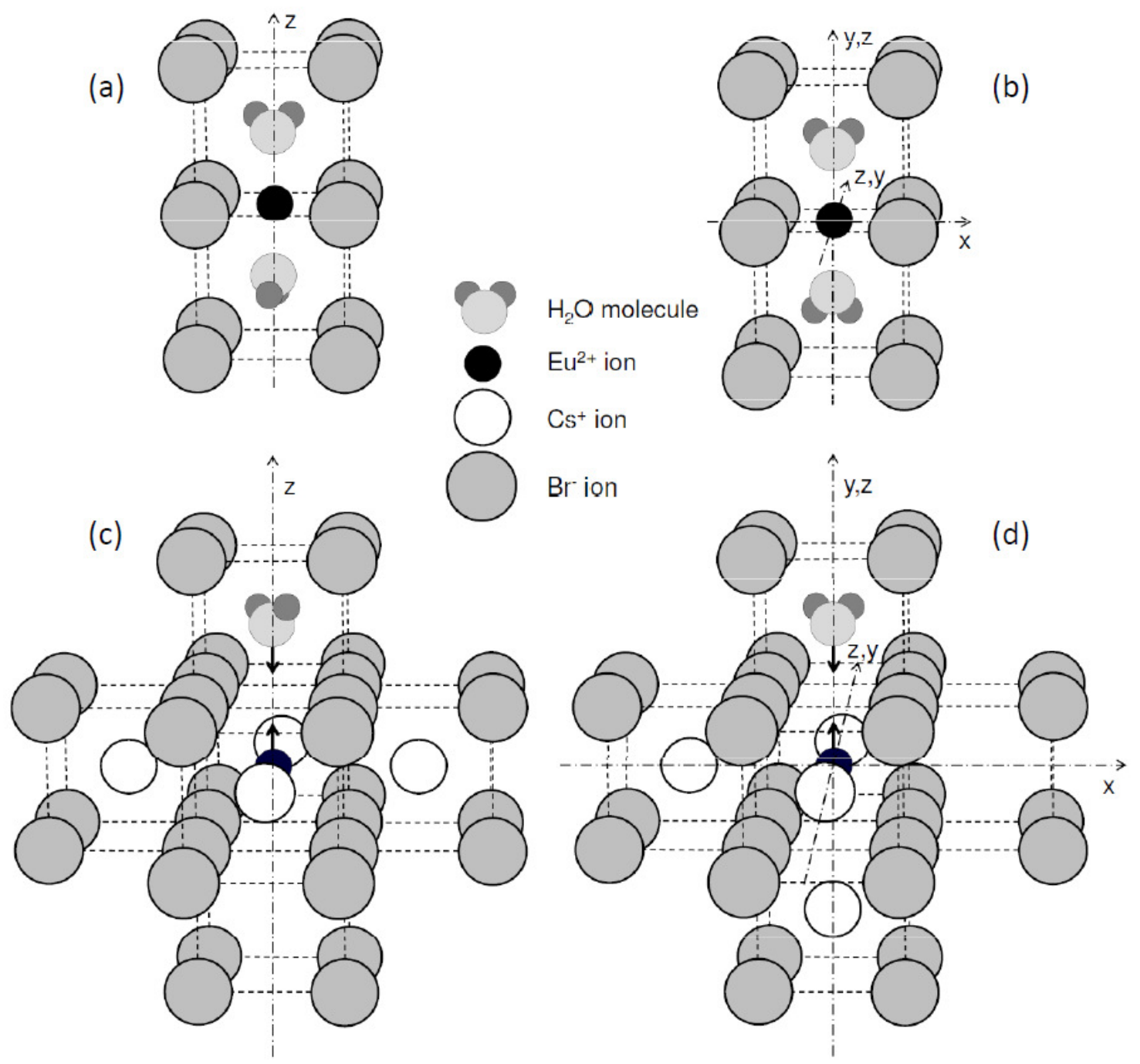

Figure 8 